\title{
Parâmetros genéticos para características de desempenho em ovinos naturalizados Sul-Mato-Grossenses
}

\section{Genetic parameters of performance traits in Sul-Mato-Grossenses naturalized sheep}

\author{
Daniele Portela de Oliveira ${ }^{1 *}$; Carlos Antonio Lopes de Oliveira ${ }^{2}$; \\ Elias Nunes Martins²; Fernando Miranda Vargas Junior³; Leonardo Oliveira Seno ${ }^{3}$; \\ Guilherme dos Santos Pinto ${ }^{4}$; Aya Sasa ${ }^{5}$; Marcos Barbosa-Ferreira ${ }^{6}$
}

\section{Resumo}

Estimações de parâmetros genéticos são importantes para o estudo de características a serem inseridas em programas de melhoramento de um grupo genético. Foram utilizadas 594 informações de pesos referentes a 211 cordeiros do grupo genético de ovinos naturalizados Sul-mato-grossenses pertencentes à Fundação Manoel de Barros, criadas no Centro Tecnológico de Ovinos (CTO) da Universidade Anhanguera-Uniderp localizado em Campo Grande-MS. A estimativa dos componentes de variância nas análises uni e bicaracter foi realizada por meio de inferência bayesiana. As estimativas de herdabilidades unicaracter variaram de 0,22 a 0,47 e bicaracter de 0,13 a 0,78 . O efeito permanente de ambiente materno foi maior no peso ao nascimento (PN) e no ganho de peso do nascimento aos 50 dias (GP0-50) em $24,2 \%$ e $19,5 \%$, respectivamente, da variação observada. As estimativas de herdabilidades, participação do efeito permanente de ambiente materno, correlações genéticas e fenotípicas indicaram que a seleção para ganho de peso do nascimento aos 90 dias implicaria em incrementos no peso aos 50 dias, peso aos 90 dias e ganho de peso dos 50 aos 90 dias (GP50-90) dos cordeiros com aumentos inexpressivos no PN e GP0-50.

Palavras-chave: Correlação, herdabilidades, peso corporal, raças nativas

\begin{abstract}
Estimates of genetic parameters are important to study characteristics that are to be included in a breeding program of a genetic group. The information of 594 weights from 211 lambs of a genetic group of naturalized Sul-mato-grossenses sheep belonging to Manoel de Barros Foundation and breeding at Centro Tecnologico de Ovinos from Anhanguera-Uniderp University was used. The estimation of variance components in unicaracter and bicaracter analysis were carried out through Bayesian inference. Estimates of heritability ranged from unicaracter analyses (0.22 to 0.47$)$ and
\end{abstract}

\footnotetext{
${ }^{1}$ Discente do Curso de Mestrado em Zootecnia, Universidade Estadual de Maringá, UEM, Maringá, PR. E-mail: dane_portela@ yahoo.com.br

2 Profs. do Dept ${ }^{\circ}$ de Zootecnia, UEM, Maringá, PR. E-mail: caloliveira@uem.br; enmartins@uem.br

${ }^{3}$ Profs. do Programa de Pós-Graduação em Zootecnia - UFGD, Dourados, MS. E-mail: fernandojunior@ufgd.edu.br; leonardoseno@ufgd.edu.br

${ }^{4}$ Médico Veterinário Autônomo, Sidrolândia, MS. E-mail: guilhermepintomv@hotmail.com

${ }^{5}$ Coordenador do Programa de Pós-Graduação em Zootecnia, Universidade Estadual de Mato Grosso do Sul, UEMS, Aquidauana, MS. E-mail: aya@uems.br

${ }^{6}$ Prof. do Mestrado em Produção e Gestão Agroindustrial, Universidade Anhanguera-Uniderp, Campo Grande, MS. E-mail: profmarcosvet@gmail.com

* Autor para correspondência
} 
the bicaracter analyses $(0.13$ to 0.78$)$. The maternal environmental permanent effect was higher in birth weight and average daily gain from birth to 50 days in $24.2 \%$ and $19.5 \%$, respectively, in the observed variation. Estimates of heritability, maternal environmental permanent effect participation, phenotypic and genetic correlations indicate that selection for average daily gain from birth to 90 days would imply increases in weight at 50 days, weight at 90 days and average daily gain from 50 to 90 days of lambs with no significant increase in birth weight and average daily gain birth at 50 days.

Key words: Body weight, correlation, heritability, local breeds

\section{Introdução}

A partir do processo de colonização do Brasil foram introduzidas diversas espécies utilizadas para produção de alimentos, entre elas os ovinos (Ovis aries). Durante cinco séculos, esses animais se multiplicaram, com mínima interferência do homem, sendo fortemente influenciados pelo processo de seleção natural, adquirindo características adaptativas e de produção para as diversas regiões do país, ficando conhecidas como raças "locais" ou “crioulas" (MORAIS, 2004).

No Mato Grosso do Sul são relatadas informações acerca de um grupo genético de ovinos, denominados "pantaneiros", adaptados às condições climáticas da região (GOMES et al., 2007). Os ovinos pantaneiros são oriundos de cruzamentos entre as raças que foram trazidas pelos colonizadores portugueses e espanhóis logo após o descobrimento (MARIANTE et al., 1999). Estes animais são encontrados em fazendas mais isoladas da região, sem nenhum controle reprodutivo ou sanitário, vivendo há muitos anos praticamente sob seleção natural (VARGAS JUNIOR et al., 2011).

CRISPIM et al. (2013) estudaram por meio de marcadores microssatélites a variabilidade genética de ovinos da "raça" Pantaneira e de outras raças sete raças amplamente comercializadas no Estado do Mato Grosso do Sul. Os autores verificaram que as sete populações de ovinos estudadas podem ser consideradas geneticamente distintas e a "raça" Pantaneira foi a que apresentou maior variabilidade genética, quando comparado aos demais grupos. Este fato indica a importância da conservação dos animais Pantaneiros, que podem possuir alelos raros que são economicamente importantes para o melhoramento genético dos ovinos.

Por outro lado, o desconhecimento das características de desempenho zootécnico dos ovinos crioulos do Pantanal estimulou pesquisa de possíveis características a serem utilizadas como critério de seleção em programas de melhoramento desses animais com a finalidade de obter animais com maior peso ao desmame visando menor período de terminação em confinamento ou a pasto. Tais ações estão de acordo com a proposta relatada por Egito, Mariante e Albuquerque (2002) que visa à estruturação de programas de preservação e conservação dos grupos genéticos naturalizados, com intuito de utilização destes no sistema produtivo, agregando características de adaptação, que normalmente são de baixa herdabilidade e consequentemente pequena resposta à seleção.

Assim, com o presente estudo objetivou-se estimar os parâmetros genéticos para características de desempenho do nascimento ao desmame de ovinos naturalizados Sul-mato-grossenses "Pantaneiros".

\section{Material e Métodos}

$\mathrm{O}$ banco de dados analisado pertence à Fundação Manoel de Barros, obtido no Centro Tecnológico de Ovinos (CTO) da Universidade AnhangueraUniderp localizado em Campo Grande-MS. As informações dos pesos são referentes aos anos de 2006, 2007, 2008 e primeiro semestre de 2009.

O conjunto de dados continha 160, 93, 116, 92, 41 e 92 mensurações dos pesos ao nascimento 
(PN), aos 50 dias (P50), aos 90 dias (P90), ganho de peso do nascimento aos 50 dias (GP0-50), ganho de peso dos 50 aos 90 dias (GP50-90) e ganho de peso médio diário do nascimento aos 90 dias (GPD), respectivamente. Estes dados foram coletados de 211 cordeiros resultantes dos acasalamentos de 19 reprodutores e 129 matrizes naturalizados Sulmato-grossenses. A matriz de parentesco continha informações de 356 animais.

Foi calculado o tamanho efetivo da população $(\mathrm{Ne})$, o coeficiente de endogamia $(\Delta \mathrm{F})$ utilizando a metodologia proposta por WRIGHT (1931), apresentada por FALCONER (1981). O Ne e o coeficiente de endogamia foram calculados pelas seguintes expressões respectivamente:

$$
\frac{1}{\mathrm{Ne}}=\frac{1}{4 \mathrm{Nm}}+\frac{1}{4 \mathrm{Nf}} ; \Delta \mathrm{F}=\frac{1}{8 \mathrm{Nm}}+\frac{1}{8 \mathrm{Nf}}
$$

em que Nm é o número observado de machos e Nf é o número observado de fêmeas.

Nas análises para as estimativas dos componentes de (co)variância e parâmetros genéticos foram considerados os efeitos de sexo e os efeitos ambientais identificáveis como tipo de parto (simples ou gemelares), mês e ano de nascimento para todas características. O efeito da idade da mãe ao parto foi utilizado como uma covariável com comportamento quadrático.

Foram estimados os componentes de (co) variância e parâmetros genéticos considerando as características individualmente, em análises unicarácter (PN, P50, P90, GP0-50, GP50-90 e GPD) e a partir de análises bicaracter para as características em estudo combinadas duas a duas (PN-P50, PN-P90, PN-GPD, PN-GP0-50, PNGP50-90, P50-P90, P50-GP0-50, P50-GP50-90, P50-GPD, P90-GP50, P90-GP50-90 e P90-GPD).

Para as estimativas dos componentes de (co) variância e parâmetros genéticos utilizou-se o seguinte modelo: $\mathbf{y}=\mathbf{X} \boldsymbol{\beta}+\mathbf{Z}_{\mathbf{1}} \mathbf{a}+\mathbf{Z}_{\mathbf{2}} \mathbf{p}+\mathbf{e}$ em que $\mathbf{y}$ é o vetor de observações das características; $\boldsymbol{\beta}$ é o vetor dos efeitos ambientais identificáveis; $\mathbf{a}, \mathbf{p}$ e e são os vetores dos efeitos genéticos aditivos diretos, efeito permanente de ambiente materno e dos erros aleatórios, respectivamente. $\mathbf{X}, \mathbf{Z}_{\mathbf{1}}$ e $\mathbf{Z}_{2}$ são as matrizes de incidência dos efeitos ambientais identificáveis, genéticos aditivos diretos e permanentes de ambiente materno, respectivamente.

Nas análises foi utilizado o sistema computacional MTGSAM - Multiple Trait Gibbs Sampling to Animal Model (VAN TASSEL; VAN VLECK, 1995), que utiliza inferência bayesiana por meio do amostrador de GIBBS, permitindo a estimativa pontual dos componentes de (co) variância e parâmetros genéticos por meio das médias posteriores e estimativa por intervalo a partir dos intervalos de credibilidade (ICr) em 95\%.

Como estratégia inicial de análise, foram definidas cadeias de Gibbs de 250.000 ciclos, sendo descartados os 25.000 primeiros ciclos, retirandose amostras a cada dez ciclos, gerando, 22.500 amostras dos componentes de (co)variância.

A verificação de convergência das cadeias geradas foi feita por meio dos testes de diagnóstico de Heildelberg e Welch pelo pacote Coda (Convergence Diagnosis and output Analyses) do programa R Development Core Team (2011). Caso não fosse verificada a convergência, o tamanho das cadeias era aumentado até atingir a convergência.

\section{Resultados e Discussão}

Os valores médios observados com seus desviospadrão para peso ao nascimento (PN), peso aos 50 dias (P50), peso aos 90 dias (P90), ganho de peso do nascimento aos 50 dias (GP0-50), ganho de peso entre 50 e 90 dias (GP50-90) e ganho de peso diário do nascimento ao desmame (GPD) foram de 3,70 $\mathrm{kg}(0,82 \mathrm{~kg}), 11,55 \mathrm{~kg}(2,73 \mathrm{~kg}), 17,82 \mathrm{~kg}(3,81$ $\mathrm{kg}), 0,150 \mathrm{~kg} /$ dia $(0,102 \mathrm{~kg}), 0,160 \mathrm{~kg} / \mathrm{dia}(0,044$ $\mathrm{kg})$ e $0,147 \mathrm{~kg} /$ dia $(0,023 \mathrm{~kg})$, respectivamente. Foram observados valores de pesos semelhantes aos encontrados em ovinos criados no Nordeste do Brasil (SARMENTO et al., 2006; SOUZA et al., 2006). 
O tamanho efetivo da população $(\mathrm{Ne})$ calculado foi de 66,24 com um coeficiente de endogamia de 0,015. Segundo a FAO (1998), o tamanho efetivo da população e coeficiente de endogamia desejáveis por geração é de 50 e 0,01 , respectivamente, valores abaixo a estes são considerados críticos. O coeficiente de endogamia encontrado por Silva et al. (2010) foi de zero, para o mesmo rebanho, usando a metodologia do software ENDOG (GUTIERREZ; GOYACHE, 2005). Kesbi (2010) encontrou um Ne de 71 para um população fechada de ovinos da raça Zandi com 2.566 animais. Já Van Wyk, Fair e Cloete (2009) verificaram em um rebanho de ovinos um $\mathrm{Ne}$ de 32,68 e endogamia média de 0,16 , os autores demonstraram que baixos valores de $\mathrm{Ne}$ estão associados a altos níveis de endogamia.
Diante destes resultados foi calculado o número de filhos por reprodutor e matriz, em que se verificou que $50 \%$ das ovelhas produziram um cordeiro, cada uma, representando a metade dos cordeiros nascidos. Quando se analisou a contribuição dos carneiros na formação da população verificou-se que do total de 19 reprodutores, apenas dois deixaram mais de 25 descendentes (Tabela 1). Isto possivelmente ocorreu pelo fato dos dois reprodutores terem sido mantidos no rebanho por um longo tempo. De acordo com Van Wyk, Fair e Cloete (2009), o aumento na contribuição genética por parte de alguns indivíduos da população tende a aumentar as relações de parentesco, o que gera aumento da endogamia e reduz o tamanho efetivo da população. Assim, neste rebanho, recomenda-se controlar os acasalamentos para evitar a ocorrência de depressão por endogamia.

Tabela 1. Frequência de cordeiros por ovelha e carneiros, respectivamente.

\begin{tabular}{ccccc}
\hline \multirow{2}{*}{ Cordeiros } & \multicolumn{5}{c}{ Frequência das Ovelhas } \\
\cline { 2 - 5 } & Absoluta & Absoluta acumulada & Relativa (\%) & Relativa acumulada (\%) \\
\hline 01 & 65 & 65 & 50,0 & 50,0 \\
02 & 48 & 113 & 37,0 & 87,0 \\
03 & 14 & 127 & 11,0 & 98,0 \\
04 & 02 & 129 & 2,0 & 100,0 \\
\hline Total & 129 & - & 100,0 & - \\
\hline & & & Frequência dos Carneiros \\
\hline 01 a 08 & 09 & 09 & 47,0 & 47,0 \\
09 a 16 & 07 & 16 & 37,0 & 84,0 \\
17 a 24 & 01 & 17 & 5,0 & 89,0 \\
\hline Total 32 & 02 & 19 & 11,0 & 100,0 \\
\hline
\end{tabular}

Fonte: Elaboração dos autores.

No procedimento para as estimativas dos componentes de variância, houve indicação de convergência para todas as cadeias tanto para análises unicaracter quanto para análises bicaracter (Tabela 2).

As médias posteriores, intervalos de credibilidade e amplitude amostral para os componentes de variância para PN, GP0-50 e GPD apresentaram pequena amplitude amostral e distribuições relativamente simétricas, sugerindo que o número de observações foi suficiente para as estimativas. Porém, para as características P50, P90 e GP50-90 não foram observados o mesmo comportamento nas estimativas. 


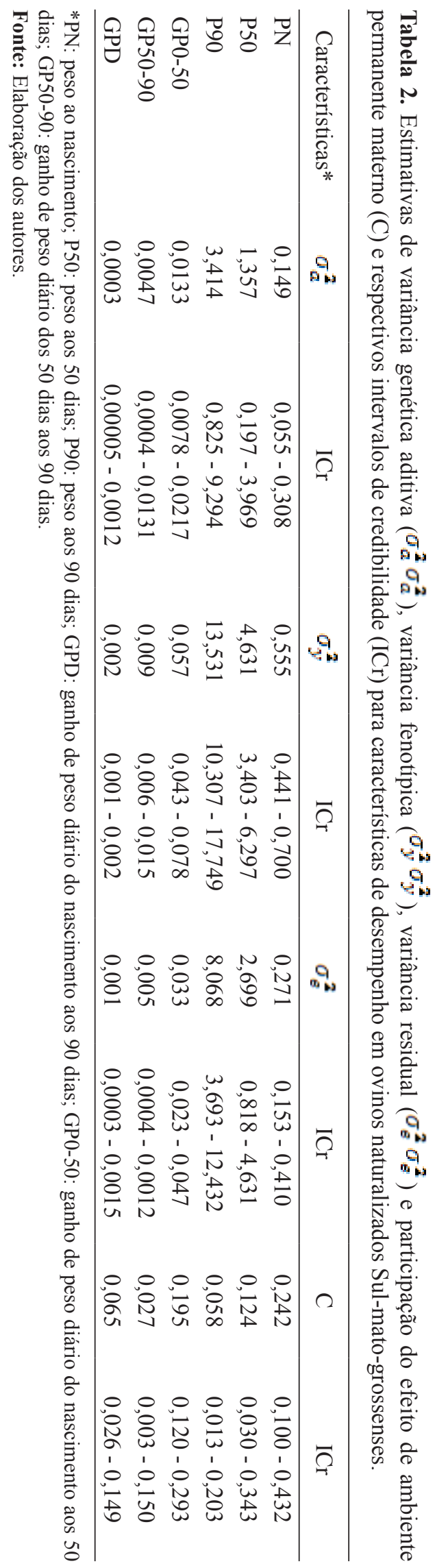


De modo geral, as estimativas das variâncias genética aditiva direta, fenotípicas e residuais em análises unicaracter para as características de peso corporal indicaram variabilidade para o grupo genético (Tabela 2).

A participação da ovelha no desempenho do cordeiro no período do nascimento ao desmame foi de maior impacto no peso ao nascimento e no ganho de peso do nascimento aos 50 dias, sendo responsável por $24,2 \%$ e $19,5 \%$, respectivamente, da variação observada. Verificou-se que com o aumento da idade do cordeiro as diferenças no ambiente permanente materno apresentaram menor participação na variação observada. Resultados semelhantes foram observados por Quesada; McManus e Couto (2002), Souza et al. (2006) e por Gholizadeh et al. (2010).

Baneh et al. (2010) afirmaram que tanto a variância genética aditiva como a variância de ambiente permanente tem participação significativa na variância observada do peso ao nascimento ao desmame em cordeiros da raça Ghezel. Segundo os mesmos autores, o cordeiro sofre maior influência da mãe neste período pela nutrição uterina, produção de leite e habilidade materna que recebem. Os resultados estimados, neste trabalho, apontam que o peso ao desmame é fracamente associado fenotipicamente ao peso ao nascimento e ao ganho de peso até os 50 dias 0,32 e 0,20, respectivamente, demonstrando que além dos efeitos genéticos aditivos existem outras causas de variação que atuam sobre estas características.

Os valores de $\mathrm{h}^{2}$ estimados apresentaram média a alta magnitude para todas as características (Tabela $3)$. As médias posteriores das herdabilidades foram de 0,$26 ; 0,28 ; 0,25 ; 0,23 ; 0,47$ e 0,22 para $\mathrm{PN}, \mathrm{P} 50$, P90, GP0-50, GP50-90 e GPD, respectivamente, para as análises unicaracter, indicando que na expressão da característica GP50-90 há maior participação dos efeitos genéticos aditivos do indivíduo comparada às outras características em que as estimativas foram de média magnitude.

Tabela 3. Estimativas de herdabilidades em análises uni e bicarácter (diagonal principal e nas linhas, respectivamente) e seus respectivos intervalos de credibilidade (abaixo) de características de desempenho para ovinos naturalizados Sul-mato-grossenses.

\begin{tabular}{lcccccc}
\hline Características* & PN & P50 & P90 & GP0-50 & GP50-90 & GPD \\
\hline \multirow{2}{*}{ PN } & $\mathbf{0 , 2 6}$ & 0,53 & 0,49 & 0,48 & 0,21 & 0,25 \\
& $\mathbf{0 , 1 0 - 0 , 5 1}$ & $0,14-0,85$ & $0,11-0,83$ & $0,08-0,86$ & $0,02-0,77$ & $0,05-0,64$ \\
P50 & 0,39 & $\mathbf{0 , 2 8}$ & 0,28 & 0,32 & 0,15 & 0,64 \\
& $0,09-0,65$ & $\mathbf{0 , 0 4 - \mathbf { 0 , 7 4 }}$ & $0,04-0,82$ & $0,03-0,85$ & $0,02-0,72$ & $0,11-0,96$ \\
P90 & 0,33 & 0,24 & $\mathbf{0 , 2 5}$ & 0,36 & 0,28 & 0,29 \\
& $0,06-0,65$ & $0,03-0,79$ & $\mathbf{0 , 0 6 - \mathbf { 0 , 6 2 }}$ & $0,06-0,68$ & $0,06-0,82$ & $0,06-0,65$ \\
GP0-50 & 0,20 & 0,16 & 0,22 & $\mathbf{0 , 2 3}$ & 0,78 & 0,21 \\
& $0,03-0,78$ & $0,09-0,25$ & $0,14-0,33$ & $\mathbf{0 , 1 4}-\mathbf{0 , 3 3}$ & $0,29-0,93$ & $0,10-0,36$ \\
GP50-90 & 0,32 & 0,31 & 0,27 & 0,22 & $\mathbf{0 , 4 7}$ & 0,13 \\
& $0,05-0,77$ & $0,08-0,62$ & $0,04-0,66$ & $0,09-0,42$ & $\mathbf{0 , 0 4 - 0 , 9 4}$ & $0,02-0,60$ \\
GPD & 0,17 & 0,32 & 0,24 & 0,58 & 0,32 & $\mathbf{0 , 2 2}$ \\
& $0,02-0,51$ & $0,10-0,76$ & $0,04-0,54$ & $0,36-0,78$ & $0,14-0,80$ & $\mathbf{0 , 0 3}-\mathbf{0 , 7 3}$ \\
\hline
\end{tabular}

*PN: peso ao nascimento; P50: peso aos 50 dias; P90: peso aos 90 dias; GPD: ganho de peso diário do nascimento aos 90 dias; GP0-50: ganho de peso diário do nascimento aos 50 dias; GP50-90: ganho de peso diário dos 50 dias aos 90 dias.

Fonte: Elaboração dos autores.

As estimativas de herdabilidade em análises - 0,64 para P50; 0,24 - 0,36 para P90; 0,16 - 0,78 bicaracter oscilaram entre 0,21 - 0,53 para PN; 0,15 para GP0-50; 0,13 - 0,32 para GP50-90 e 0,17 - 0,58 
para GPD. Observaram-se maiores diferenças nas médias posteriores e intervalos de credibilidade em análises uni e bicaracter, exceto para P90 em que os valores oscilaram de 0,25 a 0,36 com intervalos de credibilidade coincidentes. As estimativas das análises bicaracter apontaram que houve um forte impacto nas estimativas pelo acréscimo de informações. Adicionalmente, o aumento da complexidade das estruturas de covariâncias entre as características pode resultar nas diferenças observadas nas estimativas uni e bicaracter.

As estimativas de herdabilidade e participação do ambiente permanente materno no desempenho dos cordeiros indicaram que do nascimento aos 50 dias os animais sofrem maior influência dos efeitos de ambiente materno. E entre 50 e 90 dias o desempenho observado tem maior impacto do efeito genético aditivo do indivíduo, o que implica em incrementos no potencial genético para a característica de interesse.

As estimativas de herdabilidade encontradas por Gamasaee et al. (2010), Lôbo et al. (2009) e Sarmento et al. (2006) foram superiores as observadas. Todavia, Gholizadeh et al. (2010), Boujenane e Kansari (2002) e Quesada; McManus e Couto (2002) reportaram estimativas de herdabilidade inferiores as encontradas. Baneh et al. (2010) encontraram herdabilidades semelhantes as encontradas, neste trabalho.

As estimativas das correlações genéticas variaram de $-0,37$ a 0,99 . As estimativas entre $\mathrm{PN}$ e P50, PN e P90, PN e GP0-50, P50 e P90 e P90 com GPD apontam associação genética forte e positiva entre as características, indicando que a seleção para P50, P90, GP0-50 ou GPD implicaria em ganho genético no PN.

As estimativas de correlações genéticas entre PN com GPD e GP50-90, P50 e GP0-50, P90 e GP5090 foram moderadas e positivas (Tabela 4). Porém, observou-se que os ICr apresentaram valores que variam de moderadamente negativos a fortemente positivos, indicando que estas correlações genéticas podem apresentar probabilidade de ser zero.

Tabela 4. Estimativas das correlações genéticas (acima da diagonal), correlações fenotípicas (abaixo da diagonal) e seus respectivos intervalos de credibilidade (abaixo) para características de desempenho para ovinos naturalizados Sul-mato-grossenses.

\begin{tabular}{lcccccc}
\hline Características* & PN & P50 & P90 & GPD & GP0-50 & GP50-90 \\
\hline PN $(\mathrm{n}=160)$ & & 0,94 & 0,73 & 0,68 & 0,91 & 0,48 \\
& & 0,67 a 0,99 & 0,36 a 0,98 & $-0,41$ a 0,96 & 0,72 a 0,99 & $-0,61$ a 0,92 \\
P50 (n=93) & 0,45 & & 0,89 & 0,69 & 0,49 & $-0,37$ \\
& 0,26 a 0,60 & & 0,59 a 0,99 & 0,18 a 0,93 & $-0,02$ a 0,83 & $-0,86$ a 0,55 \\
P90 (n=116) & 0,32 & 0,93 & & 0,99 & 0,16 & 0,45 \\
& 0,12 a 0,50 & 0,90 a 0,95 & & 0,94 a 0,99 & $-0,29$ a 0,57 & $-0,58$ a 0,90 \\
GPD (n=92) & 0,11 & 0,85 & 0,98 & & 0,07 & 0,54 \\
& $-0,10$ a 0,31 & 0,78 a 0,90 & 0,97 a 0,98 & & $-0,40$ a 0,52 & 0,08 a 0,90 \\
GP0-50 $(\mathrm{n}=41)$ & 0,96 & 0,14 & 0,20 & 0,05 & & $-0,37$ \\
& 0,95 a 0,97 & 0,02 a 0,29 & $-0,01$ a 0,38 & $-0,22$ a 0,32 & & $-0,79$ a 0,27 \\
GP50-90 $(\mathrm{n}=92)$ & $-0,11$ & $-0,37$ & $-0,43$ & 0,76 & $-0,21$ & \\
& $-0,40$ a 0,20 & $-0,61$ a $-0,08$ & $-0,65$ a $-0,15$ & $0,66-0,84$ & $-0,48$ a 0,08 & \\
\hline
\end{tabular}

*PN: peso ao nascimento; P50: peso aos 50 dias; P90: peso aos 90 dias; GPD: ganho de peso diário do nascimento aos 90 dias; GP0-50: ganho de peso diário do nascimento aos 50 dias; GP50-90: ganho de peso diário dos 50 dias aos 90 dias.

Fonte: Elaboração dos autores. 
As estimativas de correlações genéticas entre P50 e GPD e entre GPD e GP0-50 apontaram para valores moderadamente positivos. Apesar de apresentarem valores moderados, os seus ICr foram estritamente positivos indicando que a associação entre estas características mostrou-se positiva. Todavia, para GP0-50 com P90 e GPD, as estimativas foram positivas de baixa magnitude com intervalo de credibilidade que variou de moderadamente negativo a moderadamente positivo, apontando que a seleção para GP0-50 poderia não resultar em ganhos genéticos para P90 e GPD.

Ao observarem-se as estimativas para GP5090 com P50 e GP0-50, verificou-se associação moderada e negativa com ICr fortemente negativos a moderadamente positivos, apontando que o ganho de peso dos 50 aos 90 dias dos cordeiros foi pouco associado com o P50 e com o GP0-50 dias.

Silva e Araújo (2000) estimaram correlações genéticas de 0,88 e 0,90 para P84 associada com P56 e GPD até 112 dias. Sarmento et al. (2006) observaram correlações genéticas iguais a unidade entre os pesos ao nascimento, aos 56 e 112 dias. Lôbo et al. (2009) observaram valores de correlações genéticas superiores as encontradas.

As estimativas das correlações fenotípicas variaram de $-0,43$ a 0,98 . As características mais fortemente associadas fenotipicamente entre si foram P50, P90 e GPD, indicando que cordeiros com maior peso aos 50 dias serão mais pesados aos 90 dias, e consequentemente, terão maior ganho de peso diário.

As características GP0-50 e PN possuíram forte associação fenotípica $(0,96)$ entre elas, porém, são fracamente associadas com as outras características. As estimativas de correlações fenotípicas para GP50-90 com as demais características foram de fraca a moderada, negativamente, exceto com GPD $(0,76 ; 0,66-0,84)$. Estes resultados associados com as estimativas de participação do efeito permanente de ambiente materno, herdabilidades, correlações genéticas apontam que o ganho de peso dos 50 aos 90 dias dos cordeiros foi pouco associado com o
PN e com GP0-50.

As correlações fenotípicas estimadas por McManus e Miranda (1998), Silva e Araújo (2000) e Boujename e Kansari (2002) corroboram com estes resultados. As correlações fenotípicas estimadas neste trabalho foram superiores as apresentadas por Sarmento et al. (2006).

\section{Conclusões}

As diferenças observadas no desempenho dos cordeiros até os 50 dias são mais influenciadas pelas condições oferecidas pela mãe e por outros fatores de ambiente. Porém, o desempenho do período entre os 50 dias até os 90 dias sofreu maior impacto das diferenças genéticas dos indivíduos.

As estimativas de associação genética e fenotípica aliadas aos valores de herdabilidades e participação do efeito de ambiente permanente materno na variação total indicaram que a seleção para ganho de peso diário do nascimento aos 90 dias implicaria em incrementos nos pesos aos 50 dias e 90 dias e no ganho de peso entre 50 e 90 dias dos cordeiros com aumentos inexpressivos no peso ao nascimento e no ganho de peso entre o nascimento e 50 dias.

\section{Agradecimentos}

Ao Conselho Nacional de Desenvolvimento Cientifico e Tecnológico (CNPq) e ao Centro Tecnológico de Ovinocultura da Anhaguera-Uniderp.

\section{Referências}

BANEH, H.; HAFEZIAN, S. H.; RASHIDI, A.; GHOLIZADEH, M.; RAHIMI, G. Estimation of genetic parameters of body weight traits in Ghezel sheep. AsianAustralian Journal Animal Science, Iran, v. 23, n. 2, p. 149-153, 2010.

BOUJENANE, I.; KANSARI, J. Estimates of (co) variances due to direct and maternal effects for body weights in Timahdite sheep. Animal Science, Scotland, v. 74, n. 3, p. 409-414, jun. 2002. 
CRISPIM, B. A.; GRISOLIA, A. B.; SENO, L. O.; EGITO, A. A.; VARGAS JUNIOR, F. M.; SOUZA, M. R. Genetic diversity of locally adapted sheep from Pantanal region of Mato Grosso do Sul. Genetics and Molecular Research, São Paulo, v. 12, n. 4, p. 5458-5466, nov. 2013.

EGITO, A. A.; MARIANTE, A. S.; ALBUQUERQUE, M. S. M. Programa brasileiro de conservação de recursos genéticos animais. Archivos de Zootecnia, Córdoba, v. 51, n. 193, p. 39-52, 2002.

FALCONER, D. S. Introdução à genética quantitativa. Trad. Martinho de Almeida e Silva e Jonas Carlos Silva. Viçosa: UFV, 1981. 279 p.

FOOD AND AGRICULTURE ORGANIZATION FAO. Secondary guidelines for development of national farm animal genetic resources management plans: management of small populations at risk. Rome: Food and Agriculture Organization, 1998. 215 p.

GAMASAEE, V. A.; HAFEZIAN, S. H.; AHMADI, A.; BANEH, H; FARHADI, A.; MOHAMADI, A. Estimation of genetic parameters for body weight at different ages in Mehraban sheep. African Journal of Biotechnology, Nairobi, v. 9, n. 32, p. 5218-5223, 2010.

GHOLIZADEH, M.; MIANJI, G. R.; HASHEMI, M.; HAFEZIAN, H. Genetic parameter estimates for birthand weaning weights in Raeini goats. Czech Journal of Animal Science, Czech Republic, v. 55, n. 1, p. 30-36, 2010.

GOMES, W. S.; ARAÚJO, Â. R.; CAETANO, A. R.; MARTINS, C. F.; VARGAS JUNIOR, F. M.; McMANUS, C.; PAIVA, S. R. Origem e diversidade genética da ovelha crioula do pantanal, Brasil. In: SIMPOSIO DE RECURSOS GENÉTICOS PARA AMÉRICA LATINA Y EL CARIBE, 2007, Chapingo. Anais... México: Universidad Autónoma Chapingo, 2007. 322 p.

GUTIERREZ, J. P.; GOYACHE, F. A note on ENDOG: a computer program for analysing pedigree information. Journal of Animal of Breeding and Genetics, Berlin, v. 122, n. 3, p. 172-176, 2005.

KESBI, F. G. Analyses of genetic diversity in a close population of Zandi sheep using genealogical information. Journal of Genetics, Bangalore, v. 89, n. 4, p. 479-483, dez. 2010.

LÔBO, A. M. B. O.; LÔBO, R. N. B.; PAIVA, S. R.; OLIVEIRA, S. M. P.; FACÓ, O. Genetic parameters for growth, reproductive and maternal traits in a multibreed meat sheep population. Genetics and Molecular Biology, São Paulo, v. 32, n. 4, p. 761-770, 2009.

MARIANTE, A. da S.; ALBUQUERQUE, M. do S. M.; EGITO, A. A.; McMANUS, C. Advances in the Brazilian animal genetic resources conservation programme. Animal Genetic Resources Information, Cambridge, v. 25, n. 1, p. 107-121, 1999.

MCMANUS, C.; MIRANDA, R. M. Estimativas de parâmetros genéticos em ovinos bergamácia. Revista Brasileira de Zootecnia, Viçosa, MG, v. 27, n. 5, p. 916921, 1998.

MORAIS, O. R. O melhoramento genético dos ovinos no Brasil. In: PEREIRA, J. C. C. Melhoramento genético aplicado a produção animal. 4. ed. Belo Horizonte: FEPMVZ, 2004. cap. 16, p. 358-371.

QUESADA, M.; McMANUS, C.; COUTO, F. A. D’A. Efeitos genéticos e fenotípicos sobre características de produção e reprodução de ovinos deslanados no Distrito Federal. Revista Brasileira de Zootecnia, Viçosa, MG, v. 31, n. 1, p. 342-349, 2002. Suplemento.

R DEVELOPMENT CORE TEAM. A language and environment forstatistical computing. Vienna: R: Foundation for Statistical Computing, 2011. Disponível em: <http://www.R-project.org/>. Acesso em: 15 dez. 2011.

SARMENTO, J. L. R.; TORRES, R. A.; SOUZA, W. H.; PEREIRA, C. S.; LOPES, P. S.; BREDA, F. C. Estimação de parâmetros genéticos para características de crescimento de ovinos Santa Inês utilizando modelos uni e multicaracterísticas. Arquivo Brasileiro de Medicina Veterinária e Zootecnia, Belo Horizonte, v. 58, n. 4, p. 581-589, 2006.

SILVA, D. B. S.; SENO, L. O.; GRISOLIA, A. B.; VARGAS JÚNIOR, F. M.; OLIVEIRA, C. A. L.; OLIVEIRA, D. P.; MARTINS, C. F.; PINTO, G. S. Estrutura genética dos ovinos naturalizados do Pantanal. In: CONGRESSO BRASILEIRO DE GENÉTICA, 56., 2010, Guarujá. Anais... Guarujá: Casa Grande Hotel Resort, 2010. 4 p.

SILVA, F. L. R.; ARAÚJO, A. M. de. Características de reprodução e de crescimento de ovinos mestiços Santa Inês, no Ceará. Revista Brasileira de Zootecnia, Viçosa, MG, v. 29, n. 6, p. 1712-1720, 2000.

SOUZA, J. E. R. de; OLIVEIRA, S. M. P. de; LIMA, F. de A. M.; SILVA, F. L. R.; SILVA, M. de A. S. Efeitos genéticos e de ambiente para características de crescimento em ovinos Santa Inês no Estado do Ceará. Revista Ciência Agronômica, Fortaleza, v. 37, n. 3, p. 364-368, 2006.

VAN TASSEL, C. P.; VAN VLECK D. L. A manual for use a MTGSAM. A set of FORTRAN programs to apply Gibbs sampling to animal models for variance component estimation (DRAFT). Lincoln: Department of Agriculture Research Service, 1995. 86 p. 
VAN WYK, J. B.; FAIR, M. D.; CLOETE, S. W. P. Case study: the effect of inbreeding on the production and reproduction traits in the Elsenburg Dormer sheep stud. Livestook Production Science, Reino Unido, v. 120, n. 3, p. 218-224. 2009.
VARGAS JUNIOR, F. M. de; MARTINS, C. F.; SOUZA, C. C.; PINTO, G. dos S.; PEREIRA, H. F.; CAMILO, F. R.; AZEVEDO JÚNIOR, N. P. Avaliação Biométrica de Cordeiros Pantaneiros. Revista Agrarian, Dourados, v. 4, n. 11, p. $60-65,2011$. 